\title{
Shear Stress and Atherosclerosis
}

\author{
Kyung-Sun Heo, Keigi Fujiwara, and Jun-ichi Abe*
}

\begin{abstract}
Hemodynamic shear stress, the frictional force acting on vascular endothelial cells, is crucial for endothelial homeostasis under normal physiological conditions. When discussing blood flow effects on various forms of endothelial (dys)function, one considers two flow patterns: steady laminar flow and disturbed flow because endothelial cells respond differently to these flow types both in vivo and in vitro. Laminar flow which exerts steady laminar shear stress is atheroprotective while disturbed flow creates an atheroprone environment. Emerging evidence has provided new insights into the cellular mechanisms of flowdependent regulation of vascular function that leads to cardiovascular events such as atherosclerosis, atherothrombosis, and myocardial infarction. In order to study effects of shear stress and different types of flow, various models have been used. In this review, we will summarize our current views on how disturbed flow-mediated signaling pathways are involved in the development of atherosclerosis.
\end{abstract}

\section{INTRODUCTION}

Vascular endothelial cells (ECs) form the inner lining of blood vessel wall, are directly exposed to blood flow, and serve important homeostatic functions in response to various chemical and mechanical stimuli (Berk et al., 2002; Davies, 2009; Heo et al., 2011a; 2011b; 2013; Liu et al., 2002; Shyy and Chien, 2002). In addition, ECs modulate a variety of biologic processes within the vessel wall, including active regulation of vascular tone and blood pressure through nitric oxide, endothelin, and angiotensin II (Barton et al., 2012), suppression of inappropriate activation of the coagulation system through the production of antithrombotic factors (Stern et al., 1991), and regulation of cell proliferation and angiogenesis through secretion of various growth factors and vasoactive substances (Michiels, 2003), indicating that ECs in general poses athero-protective functions.

Steady laminar flow (10-20 dyn/ $\mathrm{cm}^{2}$ ) promotes release of factors from ECs that inhibit coagulation, leukocyte diapedesis,

Aab Cardiovascular Research Institute, University of Rochester, Rochester, NY 14642, USA

*Correspondence: Jun-ichi_abe@urmc.rochester.edu

Received 3 April, 2014; accepted 7 April, 2014; published online 30 April, 2014

Keywords: chloride channel, ClC-1, myotonia congenital, skeletal muscle and smooth muscle cell proliferation while simultaneously promoting EC survival (Garin et al., 2007; Reinhart-King et al., 2008). These data suggest that steady laminar flow maintains ECs functional and athero-protective. In contrast, dysfunctional (athero-prone) ECs are readily observed in certain areas of the arterial tree, where disturbed flow, hence reduced timeaveraged shear stress, develops (Traub and Berk, 1998; Won et al., 2007). ECs in these regions have an activated, proinflammatory phenotype that is characterized by poor alignment high turnover, and being under oxidative stress (Ando and Yamamoto, 2009). Furthermore, substantial evidence supports that disturbed flow alters the profile of secreted factors and EC surface molecule expression to that favoring the development of atherosclerosis (Heo et al., 2011b; 2013; Traub and Berk, 1998), thrombosis (Koskinas et al., 2012), and EC dysfunction (Le et al., 2013), supporting that disturbed flow is athero-prone. Again, accumulating evidence suggest that steady laminar flow and disturbed flow have different effects in regulating EC function and subsequent atherosclerotic plaque formation, but lack of knowledge on the molecular mechanisms to define these two different flows effects has hindered our capability of developing anti-atheosclerotic therapy by modulating EC function. Therefore, understanding how various signal transduction pathways are affected by disturbed blood flow to cause EC dysfunction and subsequent cardiovascular diseases is critically important. This review will also highlight the emerging evidence that shear stress induced signaling in ECs is the critical early step in many cardiovascular diseases.

\section{IN VIVO AND IN VITRO MODELS FOR BLOOD FLOW STUDIES}

ECs of large arteries in vivo are constantly exposed to hemodynamic forces, such as shear stress and cyclic strain, of flowing blood. Typically, in the straight portions of a blood vessel, the flow is laminar. In these regions, ECs align and elongate parallel to the direction of flow. This alignment is thought to reduce resistance to flow and to activate signaling cascades that protect ECs from becoming dysfunctional. In contrast, blood flow within acute vessel curvatures, branch points, and bifurcations is typically disturbed, exhibiting flow reversal, separation, and overall low shear stress. As a result, ECs do not align parallel to the vessel long axis and show a less polarized shape, so-called cobblestone morphology. Because the cells do not align with the general direction of flow, their topology exposes them to greater shear stress gradients across the length of the cell, and these areas are also more prone to athe- 


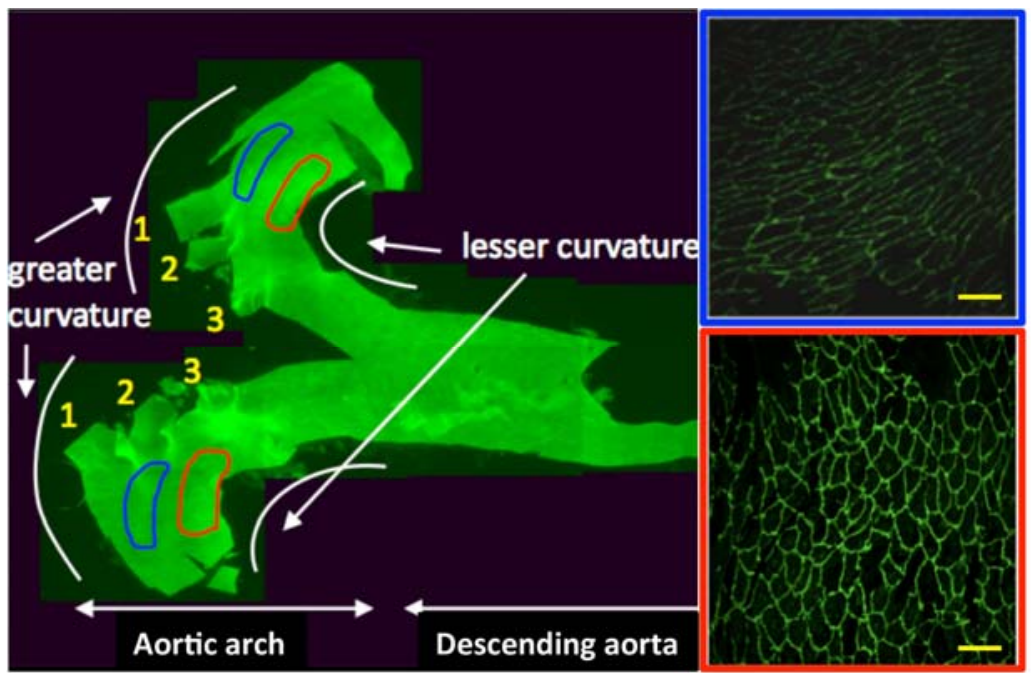

Fig. 1. En Face immunohistochemistry. The greater curvature of the aortic arch indicated by the blue circle is exposed to steady laminar flow and is protected from atherosclerosis. Regions of curvature indicated by the red circle and side branches indicated by numbers (1, 2, and 3 ) are exposed to disturbed flow and are athero-prone areas. The aorta was prepared from a 7 weeks old C57BL/6 wild type mouse. En face endothelial cells stained by anti-VE-cadherin show the general cell morphology in the steady laminar flow (blue square) and disturbed flow (red square) areas. Scale bars, $20 \mu \mathrm{m}$. rosclerosis (Heo et al., 2011a). For instance, within the carotid bifurcation, where atherosclerosis often develops, the flow separates, disrupting the laminar profile and producing disturbed streamlines (Davies, 2009; Heo et al., 2011a; Nam et al., 2009). To examine the mechanosignaling events caused by blood flow separately from those induced by chemical humoral effectors, in vitro mechanical devices have been designed to apply different types of fluid flow over a lawn of cultured ECs. To study effects of different flow patterns on ECs in vivo, different regions of the aorta are targeted for analysis. Here, we will briefly introduce En face immunochemistry used to study flow-induced mechanotransduction in ECs in vivo.

\section{EN FACE IMMUNOHISTOCHEMISTRY}

The specific areas exposed to laminar flow and disturbed flow within the aorta can be identified based on the published and generally accepted anatomical locations where such flow patterns are known to occur (Hajra et al., 2000; liyama et al., 1999; Jongstra-Bilen et al., 2006). For example, a typical laminar flow area is located in the greater curvature area and is marked as a low probability region for lesion formation (Hajra et al., 2000), which is also known as a high wall shear stress area. A disturbed flow area is the lesser curvature area where lesion formation is more prevalent (Hajra et al., 2000; liyama et al., 1999; Jongstra-Bilen et al., 2006) and is also indicated as a low wall shear stress area. EC shape outlined by anti-VE-cadherin staining can be also used to identify laminar flow areas (elongated cell shape) and disturbed flow areas (irregular cell shape) (Fig. 1).

Mouse aorta en face preparations are made in the following manner. Mice are euthanized by $\mathrm{CO}_{2}$ inhalation, and aorta is exposed by opening the abdominal cavity followed by cutting the ribs laterally to the sternum to open thoracic cavity. The arterial tree is perfused via the left ventricle with saline containing heparin (40 USPU/ml) with a 26.5-gauge needle attached to a gravity perfusion set up (pressure by height: around $100 \mathrm{~cm}$ hydrostatic pressure) to flush out blood, followed by perfusion with $4 \%$ paraformaldehyde in PBS for $10 \mathrm{~min}$. The aorta is dissected out using blunt curved scissors and forceps from the heart all the way to the iliac bifurcation including 3-5 $\mathrm{mm}$ of iliac arteries. It is important not to pull on the aorta as stretching damages the endothelium. After adipose tissues are removed, aortas are cut open longitudinally and permeabilized with PBS containing $0.1 \%$ Triton X-100 and blocked by Protein Block Serum (DAKO, X0909) for $30 \mathrm{~min}$, followed by indirect immunofluorescence staining. To obtain images shown in Fig. 1, the aorta was incubated with rat anti-VE-cadherin (endothelial cell marker) in the antibody diluent solution (DAKO, S0809) overnight. After a PBS rinse, anti-rat IgG (Alexa Fluor 488, Molecular Probes) were applied for $1 \mathrm{~h}$ at room temperature. Images were acquired using a confocal laser-scanning microscope equipped with krypton/argon/HeNe laser lines.

\section{MECHANOSENSORS OF ENDOTHELIAL SHEAR STRESS}

Elucidating the primary mechano-sensor that enables vascular ECs to distinguish between different flow profiles has been a major challenge in the field, although possible mechanosensing macromolecules and a variety of sensor cell structures have been proposed (Johnson et al., 2011) including endothelial cell-cell junction (Chiu et al., 2004; Tzima et al., 2001), heterotrimeric G-proteins (Gudi et al., 2003), caveolae (Yu et al., 2006), focal adhesions and integrins (Jalali et al., 2001), primary cilium (Nauli et al., 2013), glycocalyx (Curry and Adamson, 2012), intermediate filaments (Helmke et al., 2000), ion channels (Barakat, 1999), and the actin cytoskeleton (Osborn et al., 2006).

Davies et al. have suggested that the focal adhesion undergoes rapid remodeling in response to flow (Davies, 2009; Davies et al., 1993). In addition, fluid shear stress generated by blood flow increased accumulation of phosphotyrosin-containing protein and Src protein at the lateral cell border of endothelium, suggesting the various signaling events at the site of cell-cell junction (Kano et al., 2000). Our group reported that laminar flow induced a rapid tyrosine phosphorylation of 128 $\mathrm{kDa}$ glycoprotein in ECs, and identified it as platelet endothelial cell adhesion molecule (PECAM-1) (Osawa et al., 1997). PECAM-1 belongs to the immunoglobulin-inhibitory receptor family, which is characterized by six extracellular immunoglobulin-like domains, and also contains one trans-membrane domain and a short cytoplasmic domain. Within its short cytoplasmic domain two immunoreceptor tyrosine-based inhibitory motifs (ITIMs) exist, which are believed to have crucial role in 
initiating PECAM1-1-mediated mechano-transduction. Arguably, PECAM-1 is one of the rigorously characterized EC mechanotransducer with clear implications to cardiovascular diseases.

The crucial role of PECAM-1 as a primary "mechano-sensor" has been proposed based on the following studies by our group. First, any secondary events in signaling pathways including phosphoinositide turnover, increased cytoplasmic $\mathrm{Ca}^{2+}$, or PKC activation did not induce PECAM-1 tyrosine phosphorylation, suggesting the direct effect of mechanical force in induction of PECAM-1 tyrosine phosphorylation (Osawa et al., 1997). Second, in order to directly apply mechanical force to PECAM-1 we generated magnetic beads coated with antibodies against the PECAM-1 external domain to capture PECAM-1 on the cell surface, and then pulled the PECAM-1 eternal domain directly by applying magnetic force. We found that the only fraction of PECAM-1 bound to beads was phosphorylated and subsequently recruited SHP2 and activated ERK1/2 after magnetic force was applied (Osawa et al., 1997). Taken together these data strongly suggest that PECAM-1 is a "mechanosensor" and PECAM-1 tyrosine phosphorylation and recruitment of SHP-2 to its tyrosine phosphorylation site are the initiating processes of this mechano-transduction system. The next question is how PECAM-1 tyrosine phosphorylation is initiated, because PECAM1 contains no intrinsic enzyme activity. Our group found that Fyn is responsible to shear stress-induced PECAM-1 tyrosine phosphorylation (Chiu et al., 2008b). Based on these data we propose the following mechanism for the role of PECAM-1 in regulating shear stress-induced signaling. In the absence of shear stress, the ITIMs are covered by itself or other unidentified proteins, thus protecting them from phosphorylation. Once shear stress is applied, this protective mechanism is removed and Y713 (one of the ITIMs) is phosphorylated by Fyn, leading to SHP-2 binding and subsequently increase ERK1/2 activation (Osawa et al., 1997). Of note we could not detect Fyn kinase activation induced by shear stress, we believe that Fynmediated PECAM-1 phosphorylation is due to either a conformational change in the PECAM-1 cytoplasmic tail (Chiu et al. $2008 \mathrm{~b}$ ) or the removal of protective mechanism from phosphorylation, but the detailed mechanism of this removing process remains largely unclear.

Subsequently, Schwarts' group has proposed that a complex of vascular endothelial cell cadherin (VE-cadherin), PECAM-1, and vascular endothelial growth factor receptor 2 (VEGFR2) at cell-cell junctions form a mechanotransduction complex (Conway and Schwartz, 2012). In this study, they examined whether PECAM-1, VE-cadherin, and VEGFR2 are sufficient to transduce shear stress by transfection of encoding plasmids to COS-7 African green monkey cells and found that cells coexpressing all three proteins activated AKT and aligned in the direction of flow. Interestingly, none of the single- or doubletransfected cells showed any response to flow, suggesting that PECAM-1, VE-cadherin, and VEGFR2 represent the essential EC-specific components needed for the alignment of cells by shear stress. In addition, these proteins mainly induced flowmediated inflammatory events via activation of NF- $\mathrm{KB}$, suggesting the crucial role of PECAM-1/VE-cadherin/NEGFR2 signaling under athero-prone flow (Tzima et al., 2005). However, the role of PECAM-1 in regulating atherosclerotic plaque formation is not that simple. Based on the studies by Goel et al. (Goel et al., 2008) and Harry et al. (Harry et al., 2008), PECAM-1 promotes plaque formation in the lesser curvature of the aortic arch, which is exposed to disturbed (athero-prone) flow. In contrast, the plaque formation was increased in laminar (atheroprotective) flow area in the PECAM-1 deficient mice (Goel et al.,
2008). These data suggest that PECAM-1 can be a mechanosensor for both athero-prone and athero-protective flow. We propose that different modulating elements work with PECAM-1 under steady laminar and disturbed flow, generating different overall cellular responses in ECs under these two flow patterns. Further investigations are necessary to clarify this issue.

\section{ATHEROSCLEROSIS AND FLOW SIGNALING}

Many of the anti-atherosclerotic signals are activated by steady laminar flow, which down-regulates a number of inflammatory genes in ECs as documented by numerous transcriptional profiling studies (Boo et al., 2002; Davis et al., 2000; 2001; Fleming et al., 2005; Pi et al., 2004; Woo et al., 2008b). Roles of Gab1 and SHP2 in the shear stress-induced phosphorylation and activation of Akt and eNOS in cultured and in situ endothelial cells have been suggested (Dixit et al., 2005). Laminar shear stress-induced phosphorylation of eNOS on Ser1177 was attributed not only to the activation of the $\mathrm{Ca}^{2+} /$ calmodulindependent kinase II in bradykinin-stimulated endothelial cells but also to Akt activation in cells stimulated with vascular endothelial growth factor or hepatocyte growth factor (HGF) and protein kinase A (PKA) (Davis et al., 2000).

As explained above, endothelial dysfunction caused by disturbed flow is thought to be one of the primary culprits in the progression of atherosclerosis (Heo et al., 2011b). Besides PECAM-1/NE-cadherin/VEGFR2 signaling, we found a crucial role of SUMOylation in regulating endothelial dysfunction and atherosclerotic plaque formation under disturbed flow. SUMOylation is a unique post-translational modification akin to ubiquitination, that conjugates small ubiquitin-like proteins called small ubiquitin-like modifier (SUMO) to target proteins and affects a number of processes including the localization, degradation, binding, and activity of SUMOylated proteins (Geiss-Friedlander and Melchior, 2007). SUMOylation plays an important role in regulating variety of endothelial function including actin filament remodeling (Chang et al., 2011), migration (Chang et al., 2011), inflammation (Woo et al., 2008a), and apoptosis (Heo et al., 2011b).

In terms of flow responses, we have reported that disturbed flow activates protein kinase $\mathrm{C} \zeta(\mathrm{PKC} \zeta)$, which induces p53 SUMOylation and increases endothelial apoptosis (Heo et al., 2011b). In contrast, others and we have reported that laminar flow activates extracellular signal-regulated kinase-5 (ERK5), which plays a key role in maintaining $\mathrm{EC}$ athero-protective function via up-regulating Krüppel-like factor 2 (KLF2) expression, which leads to eNOS expression and anti-inflammatory effects (Woo et al., 2008b; Young et al., 2009). Unlike other ERK family kinases, ERK5 is not only a kinase but also a co-activator, and steady laminar flow increases PPAR $\delta$ and MEF2 transcriptional activity via its upregulation of transcriptional activity (Akaike et al., 2004; Woo et al., 2006). We found that disturbed flow increased ERK5 SUMOylation and inhibited its transcriptional activity, which down-regulated eNOS and KLF2 expression, leading to EC dysfunction and accelerating atherosclerotic plaque formation (Heo et al., 2013).

Sentrin/SUMO-specific protease 2 (SENP2) is a deSUMOylation enzyme that is important for both processing new SUMO proteins for conjugation as well as deconjugating SUMO from SUMOylated proteins (Cheng et al., 2004; Witty et al., 2010; Yeh, 2009). Although humans have six isoforms (SENP1-3 and 5-7), SENP2 specifically regulates p53 through Mdm2 de-SUMOylation, leading to apoptosis (Chiu et al., 2008a; Jiang et al., 2011). Recently, we found that reduced 


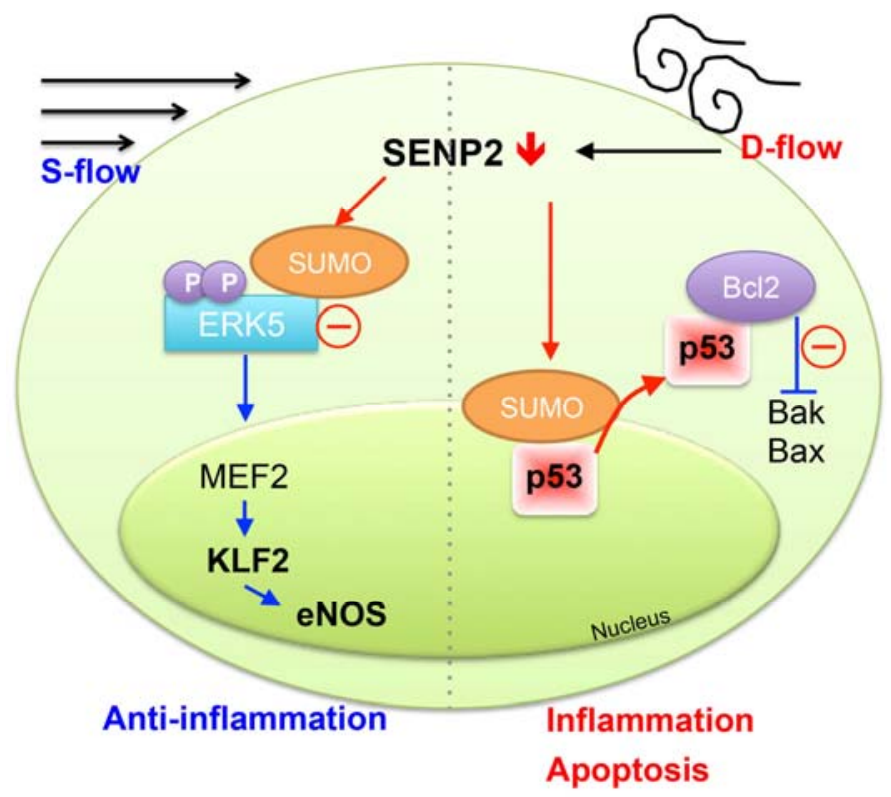

Fig. 2. Disturbed flow induces EC dysfunction via p53 and ERK5 SUMOylation. Disturbed flow down-regulates SENP2 activity, which then cause EC apoptosis via p53 SUMOylation and subsequent p53-Bcl 2 binding. On the other hand, steady laminar flow, through activation of ERK5, increases activity of MEF2/KLF2/eNOS, which are responsible for inducting antiinflammatory responses. Disturbed flow-induced downregulation of SENP2 triggers ERK5 SUMOylation and inhibits its transcriptional activity. S-flow, steady laminar flow; D-flow, disturbed flow; SUMO, small ubiquitin-like modifier.
SENP2 expression in Senp2 $2^{+-}$mice increased SUMOylation of endothelial p53 and ERK5 in ECs (Fig. 2), hence increasing EC dysfunction and inflammation (Heo et al., 2013). We then generated a double-knockout Senp $2^{+/} / L d l r^{-1}$ mouse model and determined the extent of plaque formation by high cholesterol diet. After 16 weeks on the diet, the lesion area in both aortic arch and descending aorta was significantly larger in Senp2 $2^{\text {t- }}$ $/ L d l r^{--}$than Senp2 ${ }^{++} / L d l r^{--}$mice. The lesion size in the lesser curvature where blood flow is disturbed was significantly larger in Senp2 $2^{+-} / L d l r^{-1}$ mice, suggesting a pro-atherogenic effect of disturbed flow-induced SUMOylation of proteins including ERK5 and p53. Next to support this idea, we investigated whether disturbed flow-induced EC inflammation and apoptosis via ERK5 and p53 SUMOylation and found that overexpression of ERK5 and p53 SUMOylation site mutants significantly reduced disturbed flow-induced EC inflammation and apoptosis, respectively (Heo et al., 2013). Taken together, these data strongly support a key role of SUMOylation under disturbed (athero-prone) flow and contribution of SENP2 in vascular pathology. We found that SENP2 expression was not affected by different (i.e. laminar vs disturbed) flow patterns (Heo et al., 2013). At present, it is not known how SENP2 is differentially regulated by the two flow types, and this mechanism awaits further investigation.

\section{CONCLUSION}

Although differential endothelial responses to steady laminar flow (athero-protective) and disturbed flow (athero-prone flow) have been well established and extensive studies have been done to determine the molecular mechanism of flow-initiated mecahnotrasduction, how these two types of flow revoke different responses in ECs remains largely unclear. PECAM-1 plays a key role as a sensor to initiate mechanotransduction and subsequent signaling, which is now well established. However, PECAM-1 alone cannot answer our question how laminar and disturbed flow induces different responses in ECs because both laminar and disturbed flow activate PECAM-1 signaling. PECAM-1/NE-cadherin/VEGFR2 signaling may be critical for increased EC inflammation under disturbed flow, but not under steady laminar flow. It is now important to determine the different mechanotransduction events between these two different types of flow such as SUMOylation. We should use both "top (mechanosensor) to bottom (signaling and its functional readout)" and "bottom to top" approaches to get the clear image of flow-mediated signal transduction and mechanotransduction mechanisms.

\section{ACKNOWLEDGMENTS}

This work is supported by HL-088637, HL-064839, and HL077789 to JA, 12SDG11690003 to K.S.H, and 11GRNT5850 001 to K.F.

\section{REFERENCES}

Akaike, M., Che, W., Marmarosh, N.L., Ohta, S., Osawa, M., Ding, B., Berk, B.C., Yan, C., and Abe, J. (2004). The hinge-helix 1 region of peroxisome proliferator-activated receptor gamma1 (PPARgamma1) mediates interaction with extracellular signalregulated kinase 5 and PPARgamma1 transcriptional activation: involvement in flow-induced PPARgamma activation in endothelial cells. Mol. Cell. Biol. 24, 8691-8704.

Ando, J., and Yamamoto, K. (2009). Vascular mechanobiology: endothelial cell responses to fluid shear stress. Circ. J. 73, 19831992.

Barakat, A.I. (1999). Responsiveness of vascular endothelium to shear stress: potential role of ion channels and cellular cytoskeleton (review). Int. J. Mol. Med. 4, 323-332.

Barton, M., Baretella, O., and Meyer, M.R. (2012). Obesity and risk of vascular disease: importance of endothelium-dependent vasoconstriction. Br. J. Pharmacol. 165, 591-602.

Berk, B.C., Min, W., Yan, C., Surapisitchat, J., Liu, Y., and Hoefen, R. (2002). Atheroprotective mechanisms activated by fluid shear stress in endothelial cells. Drug News Perspect. 15, 133-139.

Boo, Y.C., Hwang, J., Sykes, M., Michell, B.J., Kemp, B.E., Lum, H., 
and Jo, H. (2002). Shear stress stimulates phosphorylation of eNOS at Ser(635) by a protein kinase A-dependent mechanism. Am. J. Physiol. Heart Circ. Physiol. 283, H1819-1828.

Chang, E., Heo, K.S., Woo, C.H., Lee, H., Le, N.T., Thomas, T.N., Fujiwara, K., and Abe, J. (2011). MK2 SUMOylation regulates actin filament remodeling and subsequent migration in endothelial cells by inhibiting MK2 kinase and HSP27 phosphorylation. Blood 117, 2527-2537.

Cheng, J., Wang, D., Wang, Z., and Yeh, E.T. (2004). SENP1 enhances androgen receptor-dependent transcription through desumoylation of histone deacetylase 1 . Mol. Cell. Biol. 24, 60216028.

Chiu, Y.J., Kusano, K., Thomas, T.N., and Fujiwara, K. (2004). Endothelial cell-cell adhesion and mechanosignal transduction. Endothelium 11, 59-73.

Chiu, S.Y., Asai, N., Costantini, F., and Hsu, W. (2008a). SUMOspecific protease 2 is essential for modulating p53-Mdm2 in development of trophoblast stem cell niches and lineages. PLoS Biol. 6, e310.

Chiu, Y.J., McBeath, E., and Fujiwara, K. (2008b). Mechanotransduction in an extracted cell model: Fyn drives stretch- and flowelicited PECAM-1 phosphorylation. J. Cell Biol. 182, 753-763.

Conway, D., and Schwartz, M.A. (2012). Lessons from the endothelial junctional mechanosensory complex. F1000 Biol. Rep. 4,1.

Curry, F.E., and Adamson, R.H. (2012). Endothelial glycocalyx: permeability barrier and mechanosensor. Ann. Biomed. Eng. 40, 828-839.

Davies, P.F. (2009). Hemodynamic shear stress and the endothelium in cardiovascular pathophysiology. Nat. Clin. Pract. Cardiovasc. Med. 6, 16-26.

Davies, M.J., Gordon, J.L., Gearing, A.J., Pigott, R., Woolf, N., Katz, D., and Kyriakopoulos, A. (1993). The expression of the adhesion molecules ICAM-1, VCAM-1, PECAM, and E- selectin in human atherosclerosis. J. Pathol. 171, 223-229.

Davis, M.E., Cai, H., Drummond, G.R., and Harrison, D.G. (2000). Regulation of endothelial nitric oxide synthase (eNOS) expression by laminar shear stress (abstract). Circulation 102, II-117.

Davis, M.E., Cai, H., Drummond, G.R., and Harrison, D.G. (2001). Shear stress regulates endothelial nitric oxide synthase expression through c-Src by divergent signaling pathways. Circ. Res. 89, 1073-1080.

Dixit, M., Loot, A.E., Mohamed, A., Fisslthaler, B., Boulanger, C.M., Ceacareanu, B., Hassid, A., Busse, R., and Fleming, I. (2005). Gab1, SHP2, and protein kinase A are crucial for the activation of the endothelial NO synthase by fluid shear stress. Circ. Res. 97, 1236-1244.

Fleming, I., Fisslthaler, B., Dixit, M., and Busse, R. (2005). Role of PECAM-1 in the shear-stress-induced activation of Akt and the endothelial nitric oxide synthase (eNOS) in endothelial cells. J. Cell Sci. 118, 4103-4111.

Garin, G., Abe, J.I., Mohan, A., Lu, W., Yan, C., Newby, A.C. Rhaman, A., and Berk, B.C. (2007). Flow antagonizes TNFalpha signaling in endothelial cells by inhibiting caspasedependent PKC zeta processing. Circ. Res. 101, 97-105.

Geiss-Friedlander, R., and Melchior, F. (2007). Concepts in sumoylation: a decade on. Nat. Rev. Mol. Cell. Biol. 8, 947-956.

Goel, R., Schrank, B.R., Arora, S., Boylan, B., Fleming, B., Miura, H., Newman, P.J., Molthen, R.C., and Newman, D.K. (2008). Site-specific effects of PECAM-1 on atherosclerosis in LDL receptor-deficient mice. Arterioscler. Thromb. Vasc. Biol. 28, 19962002.

Gudi, S., Huvar, I., White, C.R., McKnight, N.L., Dusserre, N., Boss, G.R., and Frangos, J.A. (2003). Rapid activation of Ras by fluid flow is mediated by Galpha(q) and Gbetagamma subunits of heterotrimeric $\mathrm{G}$ proteins in human endothelial cells. Arterioscler. Thromb. Vasc. Biol. 23, 994-1000.

Hajra, L., Evans, A.I., Chen, M., Hyduk, S.J., Collins, T., and Cybulsky, M.I. (2000). The NF-kappa B signal transduction pathway in aortic endothelial cells is primed for activation in regions predisposed to atherosclerotic lesion formation. Proc. Natl. Acad. Sci. USA 97, 9052-9057.

Harry, B.L., Sanders, J.M., Feaver, R.E., Lansey, M., Deem, T.L., Zarbock, A., Bruce, A.C., Pryor, A.W., Gelfand, B.D., Blackman, B.R., et al. (2008). Endothelial cell PECAM-1 promotes atherosclerotic lesions in areas of disturbed flow in ApoE-deficient mice. Arterioscler. Thromb. Vasc. Biol. 28, 2003-2008.
Helmke, B.P., Goldman, R.D., and Davies, P.F. (2000). Rapid displacement of vimentin intermediate filaments in living endothelial cells exposed to flow. Circ. Res. 86, 745-752.

Heo, K.S., Fujiwara, K., and Abe, J. (2011a). Disturbed-flowmediated vascular reactive oxygen species induce endothelial dysfunction. Circ. J. 75, 2722-2730.

Heo, K.S., Lee, H., Nigro, P., Thomas, T., Le, N.T., Chang, E., McClain, C., Reinhart-King, C.A., King, M.R., Berk, B.C., et al. (2011b). PKCzeta mediates disturbed flow-induced endothelial apoptosis via p53 SUMOylation. J. Cell Biol. 193, 867-884.

Heo, K.S., Chang, E., Le, N.T., Cushman, H.J., Yeh, E.T.H., Fujiwara, K., and Abe, J.I. (2013). De-SUMOylation enzyme of sentrin/SUMO-specific protease 2 regulates disturbed flow-induced SUMOylation of ERK5 and p53 that leads to endothelial dysfunction and atherosclerosis. Circ. Res. 112, 911-923.

liyama, K., Haira, L., liyama, M., Li, H., DiChiara, M., Medoff, B.D. and Cybulsky, M.I. (1999). Patterns of vascular cell adhesion molecule-1 and intercellular adhesion molecule-1 expression in rabbit and mouse atherosclerotic lesions and at sites predisposed to lesion formation. Circ. Res. 85, 199-207.

Jalali, S., del Pozo, M.A., Chen, K., Miao, H., Li, Y., Schwartz, M.A., Shyy, J.Y., and Chien, S. (2001). Integrin-mediated mechanotransduction requires its dynamic interaction with specific extracellular matrix (ECM) ligands. Proc. Natl. Acad. Sci. USA 98, 1042-1046.

Jiang, M., Chiu, S.Y., and Hsu, W. (2011). SUMO-specific protease 2 in Mdm2-mediated regulation of p53. Cell Death Differ. 18, 1005-1015

Johnson, B.D., Mather, K.J., and Wallace, J.P. (2011). Mechanotransduction of shear in the endothelium: basic studies and clinical implications. Vasc. Med. 16, 365-377.

Jongstra-Bilen, J., Haidari, M., Zhu, S.N., Chen, M., Guha, D., and Cybulsky, M.I. (2006). Low-grade chronic inflammation in regions of the normal mouse arterial intima predisposed to atherosclerosis. J. Exp. Med. 203, 2073-2083.

Kano, Y., Katoh, K., and Fujiwara, K. (2000). Lateral zone of cell-cell adhesion as the major fluid shear stress-related signal transduction site. Circ. Res. 86, 425-433.

Koskinas, K.C., Chatzizisis, Y.S., Antoniadis, A.P., and Giannoglou, G.D. (2012). Role of endothelial shear stress in stent restenosis and thrombosis: pathophysiologic mechanisms and implications for clinical translation. J. Am. Coll. Cardiol. 59, 1337-1349.

Le, N.T., Heo, K.S., Takei, Y., Lee, H., Woo, C.H., Chang, E. McClain, C., Hurley, C., Wang, X., Li, F., et al. (2013). A crucia role for p90RSK-mediated reduction of ERK5 transcriptional activity in endothelial dysfunction and atherosclerosis. Circulation 127, 486-499.

Liu, Y., Chen, B.P., Lu, M., Zhu, Y., Stemerman, M.B., Chien, S., and Shyy, J.Y. (2002). Shear stress activation of SREBP1 in endothelial cells is mediated by integrins. Arterioscler. Thromb. Vasc. Biol. 22, 76-81.

Michiels, C. (2003). Endothelial cell functions. J. Cell. Physiol. 196, 430-443.

Nam, D., Ni, C.W., Rezvan, A., Suo, J., Budzyn, K., Llanos, A., Harrison, D., Giddens, D., and Jo, H. (2009). Partial carotid ligation is a model of acutely induced disturbed flow, leading to rapid endothelial dysfunction and atherosclerosis. Am. J. Physiol. Heart Circ. Physiol. 297, H1535-1543.

Nauli, S.M., Jin, X., AbouAlaiwi, W.A., El-Jouni, W., Su, X., and Zhou, J. (2013). Non-motile primary cilia as fluid shear stress mechanosensors. Methods Enzymol. 525, 1-20.

Osawa, M., Masuda, M., Harada, N., Bruno Lopes, R., and Fujiwara, K. (1997). Tyrosine phosphorylation of platelet endothelial cell adhesion molecule-1 (PECAM-1, CD31) in mechanically stimulated vascular endothelial cells. Eur. J. Cell Biol. 72, 229237

Osborn, E.A., Rabodzey, A., Dewey, C.F., Jr., and Hartwig, J.H (2006). Endothelial actin cytoskeleton remodeling during mechanostimulation with fluid shear stress. Am. J. Physiol. Cell Physiol. 290, C444-452.

Pi, X., Yan, C., and Berk, B.C. (2004). Big mitogen-activated protein kinase (BMK1)/ERK5 protects endothelial cells from apoptosis Circ. Res. 94, 362-369.

Reinhart-King, C.A., Fujiwara, K., and Berk, B.C. (2008). Physiologic stress-mediated signaling in the endothelium. Methods Enzymol. 443, 25-44. 
Shyy, J.Y., and Chien, S. (2002). Role of integrins in endothelial mechanosensing of shear stress. Circ. Res. 91, 769-775.

Stern, D.M., Esposito, C., Gerlach, H., Gerlach, M., Ryan, J., Handley, D., and Nawroth, P. (1991). Endothelium and regulation of coagulation. Diabetes Care 14, 160-166.

Traub, O., and Berk, B.C. (1998). Laminar shear stress: mechanisms by which endothelial cells transduce an atheroprotective force. Arterioscler. Thromb. Vasc. Biol. 18, 677-685.

Tzima, E., del Pozo, M.A., Shattil, S.J., Chien, S., and Schwartz, M.A. (2001). Activation of integrins in endothelial cells by fluid shear stress mediates Rho-dependent cytoskeletal alignment. EMBO J. 20, 4639-4647.

Tzima, E., Irani-Tehrani, M., Kiosses, W.B., Dejana, E., Schultz, D.A., Engelhardt, B., Cao, G., DeLisser, H., and Schwartz, M.A. (2005). A mechanosensory complex that mediates the endothelial cell response to fluid shear stress. Nature 437, 426-431.

Witty, J., Aguilar-Martinez, E., and Sharrocks, A.D. (2010). SENP1 participates in the dynamic regulation of Elk-1 SUMOylation. Biochem. J. 428, 247-254.

Won, D., Zhu, S.N., Chen, M., Teichert, A.M., Fish, J.E., Matouk, C.C., Bonert, M., Ojha, M., Marsden, P.A., and Cybulsky, M.I. (2007). Relative reduction of endothelial nitric-oxide synthase expression and transcription in atherosclerosis-prone regions of the mouse aorta and in an in vitro model of disturbed flow. Am. J. Pathol. 171, 1691-1704.

Woo, C.H., Massett, M.P., Shishido, T., Itoh, S., Ding, B., McClain,
C., Che, W., Vulapalli, S.R., Yan, C., and Abe, J. (2006). ERK5 activation inhibits inflammatory responses via peroxisome proliferator-activated receptor delta (PPARdelta) stimulation. J. Biol. Chem. 281, 32164-32174

Woo, C.H., Shishido, T., McClain, C., Lim, J.H., Li, J.D., Yang, J., Yan, C., and Abe, J. (2008a). Extracellular signal-regulated kinase 5 SUMOylation antagonizes shear stress-induced antiinflammatory response and endothelial nitric oxide synthase expression in endothelial cells. Circ. Res. 102, 538-545.

Woo, C.H., Shishido, T., McClain, C., Lim, J.H., Li, J.D., Yang, J., Yan, C., and Abe, J. (2008b). Extracellular signal-regulated kinase 5 SUMOylation antagonizes shear stress-induced antiinflammatory response and endothelial nitric oxide synthase expression in endothelial cells. Circ. Res. 102, 538-545.

Yeh, E.T. (2009). SUMOylation and De-SUMOylation: wrestling with life's processes. J. Biol. Chem. 284, 8223-8227.

Young, A., Wu, W., Sun, W., Benjamin Larman, H., Wang, N., Li, Y.S., Shyy, J.Y., Chien, S., and Garcia-Cardena, G. (2009). Flow activation of AMP-activated protein kinase in vascular endothelium leads to Kruppel-like factor 2 expression. Arterioscler Thromb. Vasc. Biol. 29, 1902-1908.

Yu, J., Bergaya, S., Murata, T., Alp, I.F., Bauer, M.P., Lin, M.I., Drab, M., Kurzchalia, T.V., Stan, R.V., and Sessa, W.C. (2006). Direct evidence for the role of caveolin-1 and caveolae in mechanotransduction and remodeling of blood vessels. J. Clin. Invest. $116,1284-1291$ 